\title{
Trends and predictors of asthma costs: results from a 10-year longitudinal study
}

\author{
Ngiap Chuan Tan ${ }^{1,2}$, Hai V. Nguyen², Weng Kit Lye ${ }^{2}$, Usha Sankari ${ }^{1}$ and \\ Nivedita V. Nadkarni ${ }^{2}$ \\ Affiliations \\ ${ }^{1}$ Sing Health Polyclinics, Singapore. \\ ${ }^{2}$ Duke-NUS Graduate Medical School, Singapore. \\ Correspondence: \\ Ngiap Chuan Tan, c/o Dept of Research, SingHealth Polyclinics, 167 Jalan Bukit Merah, Tower 5, \#15-10, \\ Singapore 150167. \\ E-mail: tan.ngiap.chuandsinghealth.com.sg
}

\begin{abstract}
Research on asthma costs often focuses on estimating average asthma costs. Trends in asthma costs and patterns of medication use, especially for those who have been followed up and under treatment, have received much less attention. This study's objective was to document asthma costs over time for asthma patients who are enrolled in an asthma care programme in Singapore and to identify its predictors, using a 10-year longitudinal dataset.

The study population comprised different cohorts of 939 asthma patients entering the programme at different times during 2004-2013. Average asthma costs were estimated and the trends over time examined graphically, within and across patient cohorts. Regression analyses were conducted to examine cost predictors, with a focus on the relationship between risk factors at programme enrolment and subsequent asthma costs.

The results indicate that 10-year average annual asthma cost was GBP 341 per patient. The main drivers of costs were asthma medications and consultation fees. Use of combined inhaled corticosteroid/longacting $\beta$-agonist medications increased over time, but this was accompanied by declines in controller drug use, doctor visits and total asthma drug costs. Obesity, smoking and asthma severity were the main predictors of subsequent asthma costs, especially for females.
\end{abstract}

@ERSpublications

Most asthma costs were due to drugs and consultations; the shift from controller to combination drugs was beneficial http://ow.ly/TqLcF 


\section{Introduction}

Asthma is one of the most common chronic respiratory diseases globally, affecting 334 million patients of all age groups. Asthma patients normally require long-term medical treatment of their condition and may episodically seek emergency rescue therapy at acute healthcare facilities or be hospitalised during exacerbations, resulting in absenteeism from school or work. Management of asthma thus incurs costs directly from medical consultations and therapeutics, and indirectly from the socioeconomic impact on affected individuals, their families and the community. Studies have shown that the socioeconomic burden of asthma varies across countries, as it is influenced by the structure and organisation of their respective healthcare systems and the computation of healthcare-related expenditure [1-3]. Singapore is reputed to have the second best health outcomes in the world after Japan, but health expenditures per capita to achieve this feat are less than half those of the latter [4]. Estimation of asthma cost incurred on the island state, with asthma being its most common respiratory disease, can provide an invaluable insight into its healthcare system.

In Singapore, asthma affects $10.5 \%$ of its adult multi-ethnic Asian population aged between 18 and 69 years [5]. These patients are managed largely in the walk-in, fee-for-service primary healthcare system. They receive subsidised medical treatment, including consultation fee and medications, at all public primary care clinics (polyclinics). Subsidy on asthma medications is currently limited to a few selected inhaled corticosteroid (ICS) and reliever medications in the polyclinic formulary and does not include the combined ICS/long-acting $\beta$-agonist medications (combination drugs, for short), which are indicated for patients with moderate to severe asthma based on most clinical practice guidelines.

The high cost of these drugs, despite having been proven to be effective, especially for the group of patients with severe asthma, could have deterred physicians from prescribing them and deprived patients of their access to these treatments. Thus, in 2003, a specific asthma management programme (called Reinvestment Fund (RF) programme) funded by the local health ministry was introduced to improve clinical outcomes for patients with persistent asthma in Singapore and defray the high costs of an expanded range of asthma-related medications in the formulary, which include hitherto nonsubsidised single controller and combination drugs.

The objective of this study was to characterise asthma costs over time for patients who are enrolled in the $\mathrm{RF}$ asthma care programme and investigate modifiable predictors of asthma costs. Most studies on asthma costs focus on estimating average asthma costs over a certain period of time. A decade of longitudinal data from this programme offers a unique opportunity not only to estimate the average cost of asthma treatment but also to study the trends in total asthma costs and their components over time.

\section{Methods}

\section{Reinvestment Fund programme}

The RF asthma care programme is available only in four selected polyclinics on the island, which also cater to patients with asthma who are resident in other districts. Under this RF programme, patients pay half or less of the cost of an expanded range of asthma-related medications including nonsubsidised single controller and combination drugs. Ultimately, the "reinvestment" of funds to provide additional subsidies for evidence-based effective medications to patients aims to optimise their asthma control and defray the high costs of their hospitalisation. Furthermore, patients are regularly counselled by the polyclinic healthcare team and are provided with dates and times for their subsequent visits at the end of their consultations. Defaulters of medical appointments are recalled by asthma nurse counsellors to continue their treatment at their respective polyclinic, either by telephone contact or by mail. Patients enrolled into this programme are also counselled and monitored for their asthma control by the multidisciplinary polyclinic primary care team. Their dose and type of asthma medications are adjusted by their attending physicians according to their asthma status, with less consideration for their cost.

\section{Database}

A database accompanying this RF programme was established in 2003 and recorded longitudinal data for the period 2004-2013. Programme nurses recorded patients' demographic characteristics, asthma symptoms based on Global Initiative for Asthma (GINA) guidelines, exacerbation and treatment details including frequency of consultation, rescue therapy, hospitalisation, type and dose of asthma medications, and treatment expenditures when they first entered the programme and in their subsequent visits. Data for this study are from the two participating polyclinics in the districts of Outram and Pasir Ris administered under SingHealth Polyclinics, a public primary care institution comprising a cluster of nine polyclinics located in the southern and eastern regions of the island.

\section{Study population}

The study population comprised 939 patients with physician-diagnosed asthma who entered this programme at different times during the period 2004-2013. Most of them had asthma-related symptoms due to 
suboptimal asthma control on enrolment. Some were referred from pulmonologist clinics in public hospitals, after their asthma control was established, for continuity of treatment in primary care.

\section{Cost estimation}

All costs were adjusted to the 2013 Singapore dollar using the Singapore Consumer Price Index and then converted to GBP using the 2013 average exchange rate (SGD $1=\mathfrak{E} 0.50$ ). Costs of asthma consist of direct and indirect costs and were estimated from the societal perspective. Direct costs of asthma include costs of drugs, consultation fees and asthma-related hospitalisations. Drug costs and drug dosage were recorded in the database when a patient had a prescription filled at the in-house pharmacy after each visit. Drug expenditure used in this analysis was based on unsubsidised costs to reflect realistic circumstances. Data on hospitalisations were self-reported. During each visit, patients reported to their asthma nurse counsellors the number of episodes of hospitalisation since the last visit. Based on the mean number of admission days reported by patients prior to programme enrolment, we assumed that each hospitalisation lasted for 2 days. The cost of each hospitalised day for an asthma patient is $£ 250$, based on data from the Singapore Ministry of Health [6]. Consultation costs were obtained by multiplying the number of visits with the unit cost per visit. The unit cost per consultation at polyclinic is $£ 20$ based on the nonresident rate (which reflects the costs better than the subsidised rates for Singaporean citizens and residents) [7].

Indirect costs were based on the self-reported number of school and work days lost at each visit. During each visit, patients were asked: "Since the last visit how often did you stay away from work or school due to asthma-related symptoms?" The responses were reported in ranges: " $1-2 \times$ per month", " $1-2 \times$ per week", " $\geqslant 3 \times$ per week" or "none". The mid-points of the ranges were chosen and multiplied with the time interval between the current visit and the last visit to arrive at the number of lost work days since the last visit. The number of annual lost work days was then obtained by aggregating over these visit-specific lost work days in the year. To convert lost work days into monetary value, median monthly salaries from Singapore Ministry of Manpower for different age groups were used. Specifically, median salaries for various age groups were: $£ 720$ (age 18-25 years), $£ 1430$ (age 25-29 years), $£ 1720$ (age 30-39 years), $\mathfrak{1} 1870$ (age $40-49$ years) and $£ 1880$ (age $49-65$ years) [8].

\section{Variables and definitions}

We considered two different cost measures: the annual costs and the average of annual costs. Asthma severity was classified by the 2006 GINA guidelines [9]. Since 2008, Asthma Control Test (ACT) scores have been estimated and reported in the database. Risk factors were weight status (obese if body mass index $(\mathrm{BMI}) \geqslant 27 \mathrm{~kg} \cdot \mathrm{m}^{-2}$, overweight if BMI $24-26.9 \mathrm{~kg} \cdot \mathrm{m}^{-2}$, and normal weight if BMI $<24 \mathrm{~kg} \cdot \mathrm{m}^{-2}$ ) and smoking status (current smokers, former smokers and nonsmokers). Asthma drugs used to treat patients under the programme included anti-inflammatory therapy and bronchodilators. Anti-inflammatory drugs included combination drugs (Seretide (GlaxoSmithKline, Brentford, UK) or its generic name fluticasone/ salmeterol, and Symbicort (AstraZeneca, London, UK) or budesonide/formoterol) and controller drugs (budesonide, fluticasone, beclomethasone and montelukast). Note that montelukast was classified into the controller group to be consistent with the practice in Singapore. Bronchodilators or reliever medications refer to inhaled salbutamol and oral theophylline.

\section{Cost analysis}

The 10-year average annual asthma costs were estimated and stratified by age, sex, race, severity of asthma and drug type, to study their potential differences. The differences in costs between these groups were assessed using standard statistical comparison tests (two-sample t-tests and Wilcoxon-Mann-Whitney two-sample tests as appropriate).

The average annual cost over the 10-year period may mask potential important trends over time. Furthermore, as patients entered the programme at different times, it is also possible that recently enrolled patients who were exposed to newer asthma drugs might have different cost projections from those who were enrolled earlier. Consequently, we also estimated annual costs and charted them over time for different patient cohorts. A cohort was defined as all patients entering the programme in a specific year.

To identify patient characteristics (recorded at programme enrolment) that can predict asthma costs per visit, a general estimating equation (GEE) regression was estimated using the longitudinal data at the patient-visit level. In this GEE model, we corrected for the correlations within the subjects (i.e. for each patient, costs are correlated across the visits). Patient characteristics of interest included demographics, weight status and smoking, asthma severity and drug type. To control for possible differences due to different cohorts and polyclinics, cohort and polyclinic indicator variables were included. All statistical analyses were performed using STATA version 13 (Stata Corp, College Station, TX, USA). 


\section{Results}

\section{Sample characteristics}

The study population consisted of 939 unique patients. These patients entered the programme at different times, as shown in the flowchart in online supplementary figure S1. The cohorts entering in 2005 and 2006 were of the largest size. The average dropout rate was about 6\% per year. Most of the dropouts were patients who were discharged to other healthcare providers as their asthma stabilised. Other dropouts were defaulters who were lost in follow-up and did not return. There were 18 deaths (of which two deaths were asthma related) over the study period. As the size of the cohort entering in 2004 was small (19 patients), this cohort was excluded from our subsequent cohort analysis.

Table 1 displays the characteristics of all 939 patients at their first visit. Characteristics are reported for the total sample, with an additional breakdown into children (age <16 years), adults (age 16-64 years) and the elderly (age $\geqslant 65$ years). The average age of the study population was 46 years. Chinese accounted for nearly $60 \%$, followed by Malay (20\%), Indian (14\%) and others (6\%). About half of the patients had intermittent or mild persistent asthma based on GINA 2006 classification (steps 1 and 2) and the other half had moderate to severe persistent asthma (GINA steps 3-4). There were no significant differences in terms of severity of asthma in patients who enrolled in different years (supplementary table S1). The prevalence of severe asthma (GINA step 4) was highest among elderly patients (19\%) followed by adults (16\%). The prevalence of being overweight or obese was high, accounting for more than half of the sample ( $31 \%$ obese and $20 \%$ overweight). Adults had the highest proportion with obesity (43\%), followed by the elderly (29\%) and children (6.1\%). Smoking prevalence was relatively low (7\%).

Nearly two-thirds of the sample (62\%) was given combination drugs at their first visit. The uptake rate increased with age: $45 \%$ in children, $65 \%$ in adults and $67 \%$ in elderly patients. Compared with the combination drugs, use of controller drugs was less popular for adults and the elderly (43\% and $34 \%$, respectively) but slightly more prevalent for children (50\%). The proportions of patients on controller and on combination therapy totalled over $100 \%$ because there were patients who were on both combination therapy and leukotriene receptor antagonist (i.e. montelukast). The prevalence of bronchodilator use was higher for children (60\%) than for adults and the elderly (55\% each).

\section{0-year average annual costs}

Table 2 reports the estimates for 10-year average annual asthma costs and their breakdown by sex, age and race. The average annual cost of asthma per patient was $£ 341$, of which $93 \%$ was direct costs ( $£ 318$ ) and $7 \%$ indirect costs $(£ 23)$. The largest component in direct costs was drug costs, accounting for two-thirds of

\begin{tabular}{|c|c|c|c|c|}
\hline & Whole sample & Child & Adult & Elderly \\
\hline Patients n & 939 & 179 & 531 & 229 \\
\hline Age years & $46.2 \pm 23.3$ & $8.5 \pm 4.4$ & $47.5 \pm 12.1$ & $72.8 \pm 6.3$ \\
\hline Male & 44.5 & 64.2 & 41.4 & 36.6 \\
\hline Chinese & 60.1 & 50.3 & 56.7 & 76.2 \\
\hline Indian & 14.1 & 8.9 & 16.8 & 11.5 \\
\hline Malay & 20.2 & 33.5 & 21.5 & 7.0 \\
\hline Other races & 5.6 & 7.3 & 5.1 & 5.3 \\
\hline Current smoker & 6.8 & 1.1 & 10.4 & 3.1 \\
\hline Former smoker & 11.1 & 3.9 & 12.2 & 14.1 \\
\hline Never-smoker & 82.1 & 95.0 & 77.4 & 82.8 \\
\hline Obese & 33.8 & 8.9 & 42.9 & 30.6 \\
\hline Overweight & 22.1 & 16.7 & 22.6 & 24.7 \\
\hline Normal weight & 44.1 & 74.4 & 34.5 & 44.7 \\
\hline GINA 1 & 26.5 & 21.2 & 25.0 & 34.1 \\
\hline GINA 2 & 25.3 & 30.7 & 25.5 & 20.8 \\
\hline GINA 3 & 32.3 & 36.3 & 33.3 & 26.5 \\
\hline GINA 4 & 15.9 & 11.7 & 16.3 & 18.6 \\
\hline Uptake of combination drugs" & 61.8 & 44.7 & 65.2 & 67.4 \\
\hline Uptake of controller drugs ${ }^{7}$ & 42.2 & 50.8 & 42.7 & 34.4 \\
\hline Uptake of bronchodilators $^{+}$ & 56.4 & 60.3 & 55.7 & 55.1 \\
\hline
\end{tabular}

Data are presented as mean \pm SD or \%, unless otherwise stated. GINA: Global Initiative for Asthma. \#: fluticasone/salmeterol and budesonide/formoterol; ": including budesonide, fluticasone, beclomethasone and montelukast; ${ }^{+}$: including theophylline and salbutamol. 


\begin{tabular}{|c|c|c|c|c|c|c|c|c|c|}
\hline Total costs & $341 \pm 239$ & $315 \pm 230$ & $362 \pm 245$ & $194 \pm 150$ & $369 \pm 248$ & $392 \pm 234$ & $347 \pm 239$ & $341 \pm 240$ & $319 \pm 241$ \\
\hline Direct costs & $318 \pm 207$ & $293 \pm 192$ & $338 \pm 216$ & $192 \pm 147$ & $328 \pm 192$ & $392 \pm 234$ & $327 \pm 212$ & $319 \pm 200$ & $292 \pm 185$ \\
\hline Drug cost & $221 \pm 162$ & $203 \pm 159$ & $235 \pm 163$ & $104 \pm 86$ & $238 \pm 155$ & $270 \pm 180$ & $234 \pm 171$ & $224 \pm 144$ & $192 \pm 140$ \\
\hline Cost of hospitalisation & $28 \pm 91$ & $24 \pm 82$ & $31 \pm 97$ & $26 \pm 98$ & $22 \pm 79$ & $43 \pm 107$ & $22 \pm 79$ & $25 \pm 117$ & $35 \pm 101$ \\
\hline
\end{tabular}

Data are presented as mean \pm SD unless otherwise stated. All costs are given in GBP.

the direct costs $(£ 221)$. Costs of hospitalisation were small ( $£ 28$ or $9 \%$ of direct costs). Average annual consultation costs were $£ 69$ per patient, equivalent to about four visits per year. Indirect costs (measured by lost work days converted into monetary value) were modest ( $£ 23$ ).

The total costs were higher for female than male patients ( $\$ 362$ versus $£ 315$ ). This cost difference was mostly concentrated in direct costs. For indirect costs, the difference was not statistically significant. Asthma costs increased with age for direct costs, and indirect costs were higher for adults than for children. By race, Chinese patients incurred highest costs (£347), followed by Indian (£341) and Malay (£319).

\section{Trends in costs}

Trend in annual costs

Annual asthma costs, both the totals and their breakdown into direct and indirect costs, are displayed in figure 1. The graph reveals that direct costs accounted for the majority of the total costs. Both total cost and its main component, i.e. direct cost, showed visible declining trends, except for a small increase in 2006. Indirect costs were quite stable, except for an increase in 2013.

\section{Trend in annual costs by cohort}

Figure 2 plots annual asthma costs by cohort. For each cohort, the first year's cost is lower than the second year's because not all patients enrolled in the programme at the beginning of the first year. Except for the cohort entering in 2005, whose annual total costs trended downwards over time, the costs did not decline or even increased slightly over time for other cohorts. However, the cost trajectories of the more recent cohorts lie below those of the earlier cohorts. This explains why the annual total costs in figure 1, which only averaged the costs in a given year and ignored the cohort differences, showed a general declining trend.

\section{Trend in annual cost components by cohort}

To further understand the trends in annual costs by cohort, we dissected the costs into drug costs (the main component of direct costs) and indirect costs. As annual drug costs depended on the type of drugs

FIGURE 1 Annual asthma costs: total, direct and indirect costs. All costs are given in GBP.

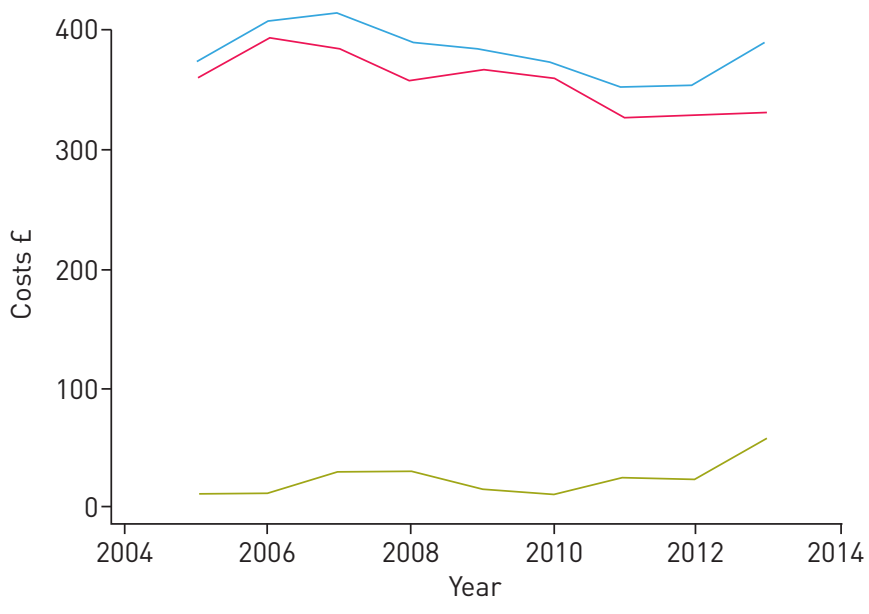

Total costs - Indirect costs 


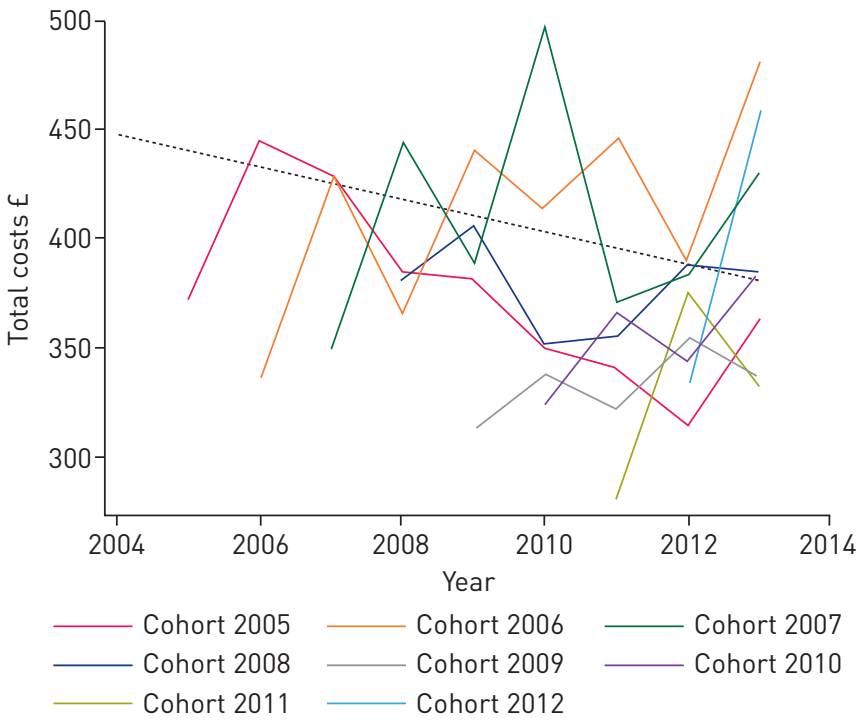

FIGURE 2 Annual total asthma costs by cohort. The dashed line indicates the linear regression line. All costs are given in GBP.

prescribed in each visit and visit frequency, we also examined the trends in the use of combination and controller drugs and in the number of visits over time.

Trend in annual asthma drug costs and indirect costs by cohort

Figure 3 displays drug costs (figure 3a) and indirect costs (figure 3b) over time for each of the cohorts. For drug costs, while the year-to-year variation became obvious, two notable features emerged. First, annual drug costs declined over time within each cohort. Secondly, recent cohorts incurred lower drug costs than the previous cohorts. Meanwhile, indirect costs tended to increase over time, especially for cohorts that entered the programme in earlier years.

Trend in annual use prevalence of combination and controller drugs by cohort

Figure 4 reveals the trend in uptake rates of combination and controller drugs over time. Uptake of combination drugs increased over time within and across the cohorts. The increase in combination drug uptake corresponded with a sharp decline in the controller drugs over time. The recent cohorts had lower uptake of controller drugs than the earlier cohorts.
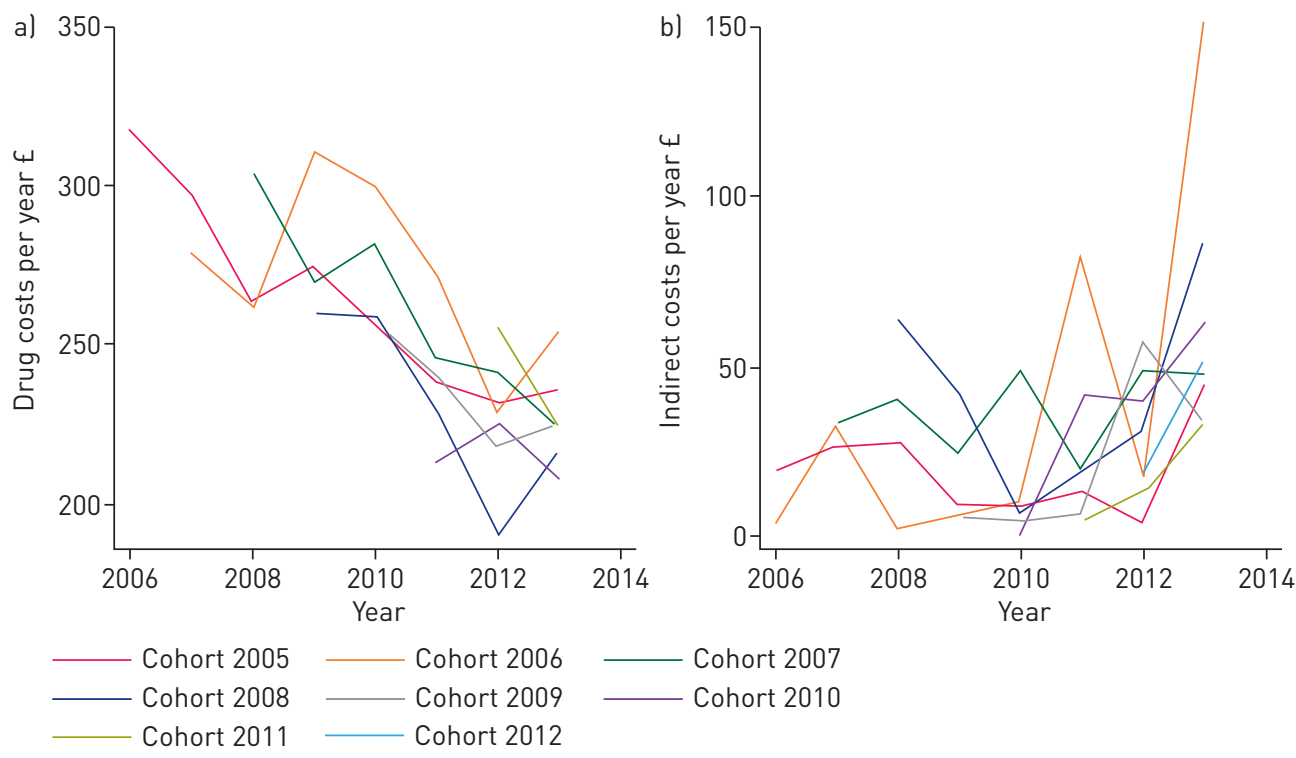

FIGURE 3 Annual asthma a) drug costs and b) indirect costs by cohort. All costs are given in GBP. 
a)

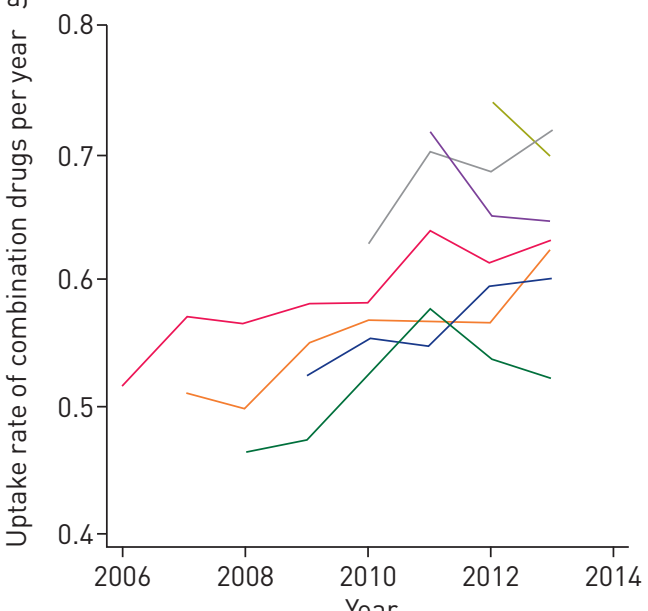

Year

b)

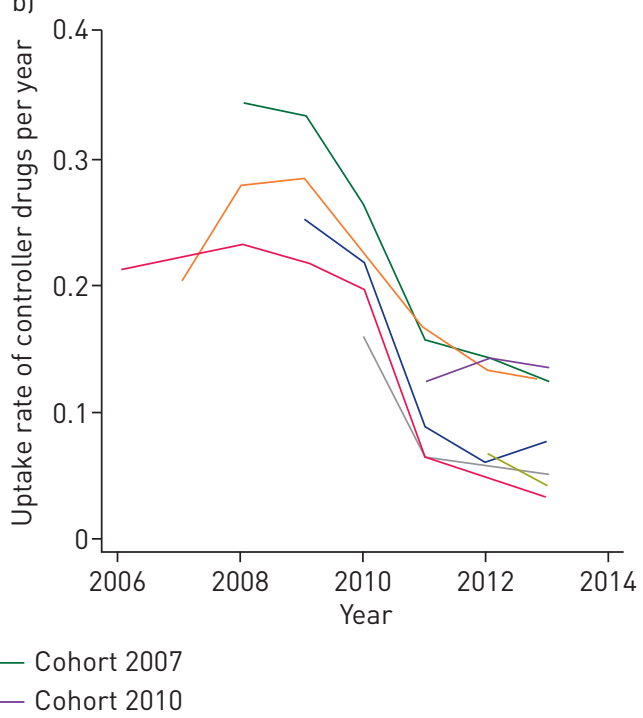

FIGURE 4 Annual uptakes of a) combination drugs and b) controller drugs by cohort.

Trend in annual number of visits by cohort

Trend in annual number of clinic visits is reported in figure 5. Recent cohorts tended to visit clinics less often than patients who entered the programme in earlier years. Within each of the cohorts, the number of visits declined over time. The decline was particularly prominent for earlier cohorts.

\section{Predictors of asthma costs}

Table 3 reports the results from the GEE regression model, exploring the associations between patient characteristics recorded in the first visit and asthma costs per visit. Estimates are reported for the entire sample and stratified by sex.

There are a number of notable results. Obese asthma patients incurred higher asthma costs than normal weight asthma patients. Specifically, in each visit, obese asthma patients paid $£ 11$ higher than their normal weight counterparts. The difference was higher for females than for males (£13 versus $£ 9$ ). There was no statistically significant difference in asthma costs between overweight patients and normal weight patients.

Smoking status also predicted the future costs. Those who reported being current smokers incurred higher costs in later years than nonsmokers. The association was stronger for females than males. Quitting smoking was beneficial, as there was no difference in asthma costs between former smokers and

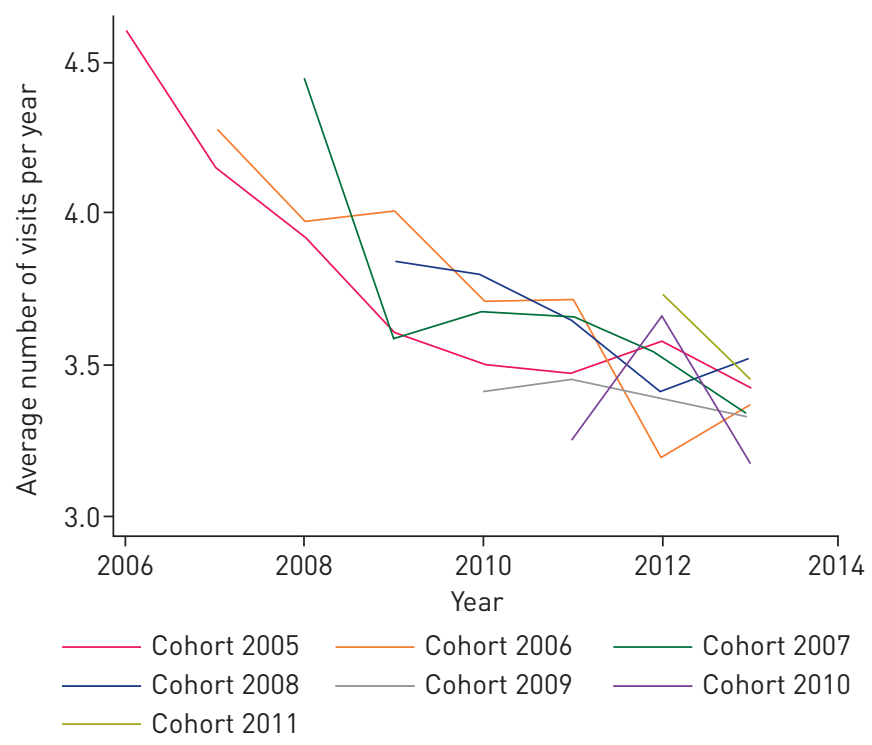

FIGURE 5 Annual number of clinic visits by cohort. 
TABLE 3 Predictors of asthma cost per visit

\begin{tabular}{lccc} 
& Total & Male & Female \\
\hline Patient visits n & 14548 & 5939 & 8609 \\
Combination drugs in first visit & $71.4(3.6)^{* * *}$ & $75.4(5.1)^{* * *}$ & $68.9(4.9)^{* * *}$ \\
GINA 2 & $8.1(4.0)^{* *}$ & $12.1(5.4)^{* *}$ & $4.7(5.9)$ \\
GINA 3 & $6.2(3.8)$ & $6.1(5.5)$ & $6.6(5.2)$ \\
GINA 4 & $12.5(4.4)^{* * *}$ & $8.3(6.2)$ & $15.5(6.0)^{* *}$ \\
Current smoker & $24.7(5.1)^{* * *}$ & $19.1(5.4)^{* * *}$ & $34.4(9.9)^{* * *}$ \\
Former smoker & $2.0(4.6)$ & $0.1(4.7)$ & $-1.5(10.0)$ \\
Obese & $11.2(3.2)^{* * *}$ & $9.1(4.2)^{* *}$ & $13.3(4.6)^{* * *}$ \\
Overweight & $0.5(3.9)$ & $-2.9(5.4)$ & $2.6(5.4)$ \\
Age & $0.1(0.1)^{*}$ & $0.3(0.1)^{* * *}$ & $0.0(0.1)$ \\
Male & $-3.2(3.2)$ & & \\
Malay & $-1.0(4.0)$ & $0.7(5.4)$ & $-2.8(5.6)$ \\
Indian & $4.6(4.2)$ & $14.5(5.2)^{* * *}$ & $-5.9(6.6)$ \\
Other races & $-7.5(6.1)$ & $-7.6(8.0)$ & $-8.8(8.9)$ \\
\hline
\end{tabular}

Results from the general estimating equation regression model, indicating difference in total asthma costs per visit, compared with reference categories, in GBP. Standard errors in parentheses. Cohort and polyclinic dummies were included to control for differences across cohorts and polyclinics; normal weight, nonsmokers and Chinese are excluded categories. GINA: Global Initiative for Asthma. ${ }^{*}: p<0.1 ;{ }^{* *}: p<0.05 ;{ }^{* * *}: p<0.01$.

never-smokers. Patients who started with combination drugs incurred $£ 71$ more costs than those who started with other drugs. This cost difference was statistically significant after adjusting for several factors, including asthma severity and demographic and risk factors.

Asthma severity was also a statistically significant predictor of costs. There was an age gradient in costs, with older patients having higher costs. There was, however, no difference in asthma costs across race and sex, after adjusting for other factors in the regression.

\section{Discussion}

This study characterised the costs of asthma incurred by enrollees, as well as the trends in cost, in a current asthma management programme that has been operational for 10 years. Average annual asthma costs were estimated to be $£ 341$ per patient under the programme. The main drivers of these costs were asthma drug costs and consultation fees. Hospitalisation only accounted for a small proportion of the total costs. This is different from several studies in the literature that found hospitalisation to be the most important cost driver of direct costs. This might suggest that the programme has been effective at helping patients to control their asthma symptoms and stay out of hospitals. It is also possible that most asthma exacerbations in Singapore are managed at general practitioner clinics or polyclinics, while most asthma exacerbations are treated in emergency units in other countries. Our estimate of $£ 341$ annually per patient in Singapore is in the lower-middle range reported in the literature. For example, in the Asia-Pacific region, annual per-patient direct costs ranged from $£ 69$ for Malaysia to $£ 646$ for Hong Kong [3].

Our analysis of asthma costs over time revealed a number of interesting patterns. First, while the annual asthma costs averaged across the cohorts declined slightly over time, the annual asthma costs for each cohort of patients increased slightly over time. The former finding is consistent with a number of studies in the literature $[10,11]$. The latter finding, however, is unique to our study. This suggests that lumping all cohorts of asthma patients together to estimate the average costs might provide misleading cost estimates, especially when the interest is in the cohort-specific cost estimates. Another notable finding is that there was a higher uptake of combination drugs over time.

The declining asthma drug costs over time warrant an explanation. As we have shown, the number of visits declined over time, which could have translated into lower costs of consultations and drugs. There is also evidence that the dosage of expensive combination drugs has declined over time. Specifically, the budesonide/formoterol dosage declined by $7 \mu \mathrm{g}$ per visit on average, while fluticasone/salmeterol dosage per visit exhibited no change over time (supplementary table S2). A decline in the number of visits together with a declining dosage of expensive combination drugs over time are consistent with the finding of an increasing proportion of asthma patients with ACT score of $\geqslant 20$ (supplementary figure S2). Combined together, it is possible that the patients' asthma has become better controlled and managed over time and the costs were reduced accordingly. 
Our analysis also indicated that a number of patient characteristics on enrolment could predict asthma costs in later years, including asthma severity, type of drugs prescribed, age, and their risk behaviour profile. We showed that asthma patients who were obese at enrolment had significantly higher average annual costs than normal weight patients, especially for female patients. This finding contributes to an emerging literature that studies the implication of obesity for asthma [12, 13]. Smoking significantly increased the costs of asthma while quitting smoking appeared to be beneficial, with the asthma costs of former smokers being similar to those of nonsmokers.

Our study has a number of limitations. First, the direct costs may be underestimated as there were no data on interaction of enrolled patients with other healthcare providers outside the two polyclinics in the programme. However, given the subsidised drug attraction of the programme, the number of patient consultations with other health providers, if any, was likely to be small. Similarly, indirect costs were probably underestimated as we did not quantify costs pertaining to children's absence from school due to asthma symptoms and the costs of their caregivers, or loss of productivity for working patients. Secondly, the estimated costs were specific to enrolled patients under this programme. However, comparison suggests that our patient population approximates asthma patients who are treated with usual care in the Singapore public primary care system. Thirdly, there was no control group in our study. Finally, any cost estimation should be assessed relative to its asthma outcomes, especially from a longitudinal perspective. Although our analysis touched upon this issue, a more comprehensive analysis is warranted. That is a topic for future research.

To conclude, this study demonstrated that most of the costs of asthma were related to drugs and consultations in the primary care outpatient setting. Our analysis also indicated that the shift from controller drugs to combination drugs over time was beneficial as it helped reduce asthma drug costs and led to better asthma control.

\section{References}

1 Bahadori K, Doyle-Waters MM, Marra C, et al. Economic burden of asthma: a systematic review. BMC Pulm Med 2009; 9: 24

2 Barnett SB, Nurmagambetov TA. Costs of asthma in the United States: 2002-2007. J Allergy Clin Immunol 2011; 127: $145-152$.

3 Lai CK, Kim YY, Kuo SH, et al. Cost of asthma in the Asia-Pacific region. Eur Respir Rev 2006; 15: 10-16.

4 The Economist Intelligence Unit Healthcare. Health outcomes and cost: a 166-country comparison. London, Economist Intelligence Unit, 2014. Available from: www.eiu.com/public/topical_report.aspx?campaignid= Healthoutcome2014

5 Epidemiology and Disease Control Division, Singapore Ministry of Health. National Health Survey 2010. Available from: www.moh.gov.sg/content/moh_web/home/Publications/Reports/2011/national_health_survey2010.html

6 Singapore Ministry of Health. Hospital Bill Sizes: Asthma. www.moh.gov.sg/content/moh_web/home/costs_and_ financing/HospitalBillSize/asthma.html Date last accessed: December 28, 2014

7 SingHealth Polyclinics. Patient Care: Fees. 2014 data. http://polyclinic.singhealth.com.sg/PatientCare/Fees/Pages/ Home.aspx Date last accessed: December 28, 2014.

8 Income, earnings and wages. In: Ministry of Manpower. Singapore Yearbook of Manpower Statistics, 2013. Singapore, Ministry of Manpower, 2013.

9 Global Initiative for Asthma. Global Strategy for Asthma Management and Prevention Revised 2006. GINA, 2006. Available from: www.ginasthma.org/local/uploads/files/GINA_Report_072007_1.pdf

10 Weiss KB, Sullivan SD, Lyttle CS. Trends in the cost of illness for asthma in the United States, 1985-1994. J Allergy Clin Immunol 2000; 106: 493-499.

11 Bedouch P, Sadatsafavi M, Marra CA, et al. Trends in asthma-related direct medical costs from 2002 to 2007 in British Columbia, Canada: a population based-cohort study. PLoS One 2012; 7: e50949.

12 Boulet LP. Asthma and obesity. Clin Exp Allergy 2013; 43: 8-21.

13 Mohanan S, Tapp H, McWilliams A, et al. Obesity and asthma: pathophysiology and implications for diagnosis and management in primary care. Exp Biol Med 2014; 239: 1531-1540. 